\title{
Glutamyl-Prolyl tRNA Synthetase mRNA is Highly Expressed in Hepatocellular Carcinoma and May be Associated with Poor Prognosis
}

Jinyong Shu

Southern Medical University

Yi Gao ( $\square$ drgaoy@126.com )

Southern Medical University https://orcid.org/0000-0003-3525-0133

Guifeng Zhang

Chinese Academy of Sciences

Pan Luo

Yueyang First People's Hospital

\section{Primary research}

Keywords: Hepatocellular carcinoma, bioinformatics, glutamyl-prolyl tRNA synthetase, aminoacyl-tRNA synthetase

Posted Date: September 9th, 2021

DOl: https://doi.org/10.21203/rs.3.rs-817259/v1

License: (c) (i) This work is licensed under a Creative Commons Attribution 4.0 International License. Read Full License 


\section{Abstract}

\section{Background}

Although glutamyl-prolyl tRNA synthetase (EPRS) mRNA is overexpressed and plays an important role in most tumors, its role in the development and progression of hepatocellular carcinoma (HCC) remains unclear.

\section{Methods}

The expression of EPRS in tumor and adjacent tissues was queried using TIMER and The Cancer Genome Atlas. The results were validated using the real-time reverse transcription polymerase chain reaction on RNA extracted from tumor and adjacent non-tumor samples from $10 \mathrm{HCC}$ patients.

\section{Results}

Using bioinformatics analysis, we found that EPRS mRNA was overexpressed in HCC tumor tissues, and the expression level of EPRS mRNA in The Cancer Genome Atlas database was significantly correlated with tumor size $(p=0.0010)$, histological grade $(p=0.0002)$, TNM stage $(p=0.0001)$, and vascular invasion ( $p=0.0123$ ) of HCC. The Kaplan-Meier survival analysis demonstrated that the expression of EPRS mRNA was associated with poor overall survival $(p=0.0004)$. Ten pairs of tumor and adjacent normal tissues were collected from patients with HCC, and the expression of EPRS mRNA was verified. The results showed that the EPRS mRNA level in HCC tissues was higher than that in paracancerous tissues $(p=0.0401)$.

\section{Conclusion}

Overexpression of EPRS mRNA may be associated with tumorigenesis and the progression of HCC.

\section{Background}

Liver cancer is the sixth most common malignancy worldwide, and hepatocellular carcinoma (HCC) is the most common type of liver cancer. In 2015, approximately 788,000 patients died of liver cancer [1]. Although many treatments are available, the mortality rate of HCC remains very high [2]. This is mainly due to the lack of effective and accurate treatments for this disease.

Aminoacyl-tRNA synthetases (ARSs) are an ancient class of enzymes that link specific amino acids to their cognate tRNAs to accurately decode genetic information during protein synthesis [3]. Many ARSs play important roles in various pathophysiological processes, including development, angiogenesis, tumorigenesis, obesity, and inflammation [4-8]. ARS expression profiles can serve as useful cancer prognostic tools because they correlate with the overall survival (OS) of patients with each cancer listed in the Human Protein Atlas database. Patients with high expression of ARSs usually have shorter survival times. For example, high expression of glycyl-tRNA synthetase is considered an indicator of poor 
prognosis in patients with renal, urothelial, liver, breast, and endometrial cancers, while increased expression of methionyl-tRNA synthetase in tumor areas of lung tissue is associated with poor prognosis, including shorter OS and disease-free survival.

Glutamyl-prolyl tRNA synthetase (EPRS) is a member of the ARS family that conjugates the amino acid proline to its cognate tRNA to generate prolyl-tRNA, which can then be used in protein synthesis. Collagen plays an important role in tissue fibrosis, and its main component is proline [9-11]. Previous studies reported that EPRS can promote tissue fibrosis [12-14]. HCC is closely associated with hepatic fibrosis [15]. Therefore, EPRS may be closely related to tumorigenesis of HCC. However, the role of EPRS in tumorigenesis and the progression of HCC remains unclear.

In this study, we investigated the expression of EPRS mRNA in HCC tissues through bioinformatics analysis, and its correlation with clinicopathological features and prognosis. For confirmation, we used fresh human tissue specimens to verify the differential expression of EPRS mRNA in HCC tumor and adjacent non-tumor tissues. The results demonstrated that EPRS mRNA is associated with the progression and metastasis of HCC. We expect that EPRS will be a new target for the treatment of HCC.

\section{Methods}

\section{Bioinformatics analysis}

The expression of EPRS in tumor and adjacent tissues was queried using TIMER, an online analysis tool. We found that EPRS was highly expressed in many tumors, including HCC, and its expression level in tumor tissues was significantly higher than that in adjacent normal tissues. The Cancer Genome Atlas (TCGA) (https://www.cancer.gov/about-nci/organization/ccg/research/structural-genomics/tcga) contains a wide variety of detailed data on many tumors [16]. Level 3 gene expression and clinical followup data for HCC were downloaded from TCGA database. After a series of data pre-processing steps, EPRS mRNA expression data were extracted from each sample (Additional file 1).

\section{Patients and tissue preparation}

Ten patients with HCC received surgical treatment without neoadjuvant therapy at Hunan Provincial People's Hospital from August 2019 to October 2019. Cancerous and paired normal tissues (obtained from a distance of $2 \mathrm{~cm}$ ) were collected from these patients. To extract RNA, intraoperative specimens were immediately frozen in liquid nitrogen and stored at $-80^{\circ} \mathrm{C}$. The study was approved by the Ethics Committee of Zhujiang Hospital of Southern Medical University (Approval No: 2017-gdek-004).

\section{Real-time reverse transcription polymerase chain reaction}

TRIzol reagent (Invitrogen, Carlsbad, CA, USA) was used to extract total RNA from tissues according to the manufacturer's instructions. First-strand cDNA was synthesized using $1 \mu \mathrm{g}$ of total RNA and the RevertAid $^{\text {TM }}$ H Minus First Strand cDNA Synthesis Kit (Fermentas, Burlington, ON, Canada) as described by the manufacturer. EPRS and glyceraldehyde 3-phosphate dehydrogenase (GAPDH) (internal control) 
primers were designed and their sequences were as follows: EPRS forward: $5^{\prime}-$ GCCTTCAGGGACAGTAAGCA-3', reverse: 5'-ATGAAGTTGCTGCACAAGGG-3'; GAPDH forward 5'CAATGACCCCTTCATTGACC-3', reverse 5'-GACAAGCTTCCCGTT CTCAG-3'.

\section{Statistical analyses}

SPSS software (version 20.0; IBM, Chicago, IL, USA) was used for statistical analyses. The relationship between EPRS mRNA expression and clinicopathological characteristics was analyzed using the chisquare test. Survival curves were plotted using the Kaplan-Meier method, the log-rank test was used for survival comparisons between groups, and the best cut-off value was calculated using X-TILE software. Multivariate Cox proportional hazards models were used to estimate the independent risk factors for survival. Statistical significance was set at $p<0.05$.

\section{Results}

\section{EPRS mRNA is highly expressed in most tumors, including HCC}

TIMER is a tool for visualization of TCGA data. Through this online tool, we found that EPRS mRNA was expressed in many tumor and adjacent normal tissues. In most tumors, the expression level of EPRS mRNA was higher than that in adjacent normal tissues (Fig. 1), including breast cancer, cholangiocarcinoma, colonic adenocarcinoma, lung adenocarcinoma, prostate cancer, stomach adenocarcinoma, and HCC.

\section{Correlation of EPRS mRNA expression with clinicopathological features in HCC}

The association between EPRS mRNA expression and clinicopathological features of HCC was analyzed based on TCGA data (Table 1). EPRS mRNA expression was closely correlated with tumor size ( $p=$ $0.001)$, histological grade $(p=0.0002)$, vascular invasion $(p<0.0123)$, and TNM stage $(p=0.0001)$ in patients with HCC, but there was no significant association with family history, sex, or age.

\section{Survival in relation to the EPRS mRNA level}

We extracted EPRS gene expression and survival data from TCGA database using a series of data preprocessing steps (Additional file 2). According to the optimal cut-off value (62.7) calculated by X-TILE software, all patients were divided into high and low EPRS expression groups. There were 274 patients in the low expression group and 96 in the high expression group. The Kaplan-Meier survival analysis demonstrated that the expression of EPRS was associated with OS of HCC patients (Fig. 2). Specifically, higher EPRS mRNA expression was associated with poorer OS.

\section{EPRS mRNA expression between newly collected HCC and adjacent non-tumor tissues}

To verify the EPRS mRNA expression level between HCC tumor and adjacent non-tumor tissues, we collected 10 pairs of fresh tissue samples. The expression level of EPRS mRNA in HCC tumor tissues was 
significantly higher than that in paired adjacent non-tumor tissues (Fig. 3).

\section{Discussion}

HCC is one of the most deadly tumors. This is because of the lack of an effective comprehensive treatment. Therefore, further research into the pathogenesis and progression of HCC will help in the development of new therapies or drugs. ARSs are a family of housekeeping enzymes involved in cell translation. Ribosomal RNA is the main component of ribosomes; therefore, the rate of ribosomal RNA synthesis may be associated with cancer prognosis [17].

Some ARSs show cancer-associated overexpression $[18,19]$. It is not clear whether this increase is due to an increased demand for protein synthesis by cancer cells or whether ARSs are a driver of cell transformation [17]. EPRS is an ARS [20], and some studies suggest that it is a tumor immunogen in human breast and gastric cancers [20]. It has been also reported that high expression of EPRS in estrogen receptor-positive breast cancer is associated with poor prognosis [21]. However, the role of EPRS in HCC has not yet been reported.

In our study, the expression level of EPRS mRNA in HCC was higher than that in adjacent non-tumor tissues, which was consistent with data from TCGA. These results indicate that EPRS may function as an oncogene and play an important role in HCC tumorigenesis. Based on TCGA data, we found that the EPRS mRNA expression level was associated with poor tumor differentiation, large tumor size, advanced TNM stage, vascular invasion, and shorter OS time. These results suggest that EPRS may play an important role in the progression of HCC. Consistent with this concept, some studies suggest that inhibiting the expression of EPRS can block the development of resistance to endocrine therapy in patients with hormone receptor-positive breast cancer [21]. Other studies also found that inhibiting the activity of EPRS can affect cell proliferation [22]. Therefore, EPRS may serve as a therapeutic target for HCC.

\section{Conclusions}

In summary, EPRS plays an important role in the tumorigenesis and progression of HCC and can be potentially used as a prognostic biomarker and therapeutic target for HCC.

\section{Abbreviations}

ARS, aminoacyl-tRNA synthetase; EPRS, glutamyl-prolyl tRNA synthetase; GAPDH, glyceraldehyde 3phosphate dehydrogenase; HCC, hepatocellular carcinoma; OS, overall survival; TCGA, The Cancer Genome Atlas

\section{Declarations}

1. Ethics approval and consent to participate 
The study was approved by the Ethics Committee of Zhujiang Hospital of Southern Medical University (Approval No: 2017-gdek-004).

\section{Consent for publication}

Written informed consent for publication was obtained from all participants.

\section{Availability of data and materials}

The datasets used or analyzed in the current study are available from the corresponding author upon reasonable request.

\section{Conflict of Interest}

The authors declare that the research was conducted in the absence of any commercial or financial relationships that could be construed as a potential conflict of interest.

\section{Author Contributions}

Jinyong Shu: Research concept and design, Data analysis and interpretation, Writing the article. YiGao: Critical revision of the article, Final approval of article. Guifeng Zhang: Critical revision of the article, Final approval of article. Pan Luo: Collection and/or assembly of data

\section{Funding}

This work was supported by the National Key R\&D Program of China (2018YFC1106400; 2018YFA0108200), the National Natural Science Foundation of China (31972926), the Natural Science Foundation of Guangdong Province (2014A030312013,2018A030313128), the Science and Technology Program of Guangzhou (201803010086), and the Guangdong Key Research and Development Plan (2019B020234003).

\section{Acknowledgments}

We would like to thank Editage (www.editage.cn) for English language editing.

\section{References}

1. Bray F, Ferlay J, Soerjomataram, Siegel RL, Torre LA, Jemal A. Global cancer statistics 2018 : GLOBOCAN estimates of incidence and mortality worldwide for 36 cancers in 185 countries. CA Cancer J Clin. 2018;68(6):394-424.

2. Kim DY. New systemic therapies for advanced hepatocellular carcinoma. Korean J Gastroenterol. 2019;73(1):10-5.

3. Eswarappa SM, Potdar AA, Sahoo S, Sankar S, Fox PL. Metabolic origin of the fused aminoacyl-tRNA synthetase, glutamyl-prolyl-tRNA synthetase. J Biol Chem. 2018;293(49):19148-56. 
4. Guo M, Schimmel P. Essential nontranslational functions of tRNA synthetases. Nature Chem Biol. 2013;9(3):145-53.

5. Havrylenko S, Mirande M. Aminoacyl-tRNA synthetase complexes in evolution. Int J Mol Sci. 2015;16(12):6571-94.

6. Lee SW, Cho AH, Park SG, Kim S. Aminoacyl-tRNA synthetase complexes: beyond translation. J Cell Sci. 2004;117(Pt 17):3725-34.

7. Rajendran V, Kalita P, Shukla H, Kumar AA, Tripathi T. Aminoacyl-tRNA synthetases: Structure, function, and drug discovery. Int J Biol Macromol. 2018;111(11):400-14.

8. Arif A, Terenzi F, Potdar AA, Jia J, Sacks J, China A, et al. EPRS is a critical mTORC1-S6K1 effector that influences adiposity in mice. Nature. 2017;542(7641):357-61.

9. Han HS, Lee H, You DG, Nguyen VQ, Song D-G, Oh BH, et al. Human adipose stem cell-derived extracellular nanovesicles for treatment of chronic liver fibrosis. J Cont Rel. 2020;123(121):123-5.

10. Hohenester S, Kanitz V, Kremer AE, Paulusma CC, Wimmer R, Kuehn H, et al. Glycochenodeoxycholate promotes liver fibrosis in mice with hepatocellular cholestasis. Cells. 2020;9(2):323-7.

11. El-Safy S, Tammam SN, Abdel-Halim M, Ali ME, Youshia J, Boushehri MAS, et al. Collagenase loaded chitosan nanoparticles for digestion of the collagenous scar in liver fibrosis: the effect of chitosan intrinsic collagen binding on the success of targeting. Eur J Pharm Biopharm. 2020;23(12):125-9.

12. Neenan TX, Burrier RE, Kim S. Biocon's target factory. Nat Biotechnol. 2018;36(9):791-7.

13. Song D-G, Kim D, Jung JW, Nam SH, Kim JE, Kim H-J, et al., Glutamyl-prolyl-tRNA synthetase regulates epithelial expression of mesenchymal markers and extracellular matrix proteins: implications for idiopathic pulmonary fibrosis. Front Pharmacol. 2018;9:1337.

14. Shibata A, Kuno M, Adachi R, Sato Y, Hattori H, Matsuda A, et al. Discovery and pharmacological characterization of a new class of prolyl-tRNA synthetase inhibitor for anti-fibrosis therapy. PLoS One. 2017;12(10):e0186587.

15. O'Brien C, Ramlual N, Haughey A, Nolan N, Malone DE, McCormick PA. Hepatocellular carcinoma in cystic fibrosis liver disease: a cautionary tale. QJM. 2019;112(9):693-4.

16. National Cancer Institute. The Cancer Genome Atlas Program. https://tcga-data.nci.nih.gov/tcga/. Accessed Date囚June 18, 2019

17. Kim S, You S, Hwang D. Aminoacyl-tRNA synthetases and tumorigenesis: more than housekeeping. Nature Rev Cancer. 2011;11(10):708-18.

18. Shin S-H, Kim H-S, Jung S-H, Xu H-D, Jeong Y-B, Chung Y-J. Implication of leucyl-tRNA synthetase 1 (LARS1) over-expression in growth and migration of lung cancer cells detected by siRNA targeted knock-down analysis. Exp Mol Med. 2008;40(2):229-36.

19. Vellaichamy A, Sreekumar A, Strahler JR, Rajendiran T, Yu J, Varambally S, et al. Proteomic interrogation of androgen action in prostate cancer cells reveals roles of aminoacyl tRNA synthetases. PLoS One. 2009;4(9):e7075. 
20. Line A, Slucka Z, Stengrevics A, Silina K, Li G, Rees RC. Characterisation of tumour-associated antigens in colon cancer. Cancer Immunol Immunother. 2002;51(10):574-82.

21. Katsyv I, Wang M, Song WM, Zhou X, Zhao Y, Park, S, et al. EPRS is a critical regulator of cell proliferation and estrogen signaling in ER+ breast cancer. Oncotarget. 2016;7(43):69592-605.

22. Keller TL, Zocco D, Sundrud MS, Hendrick M, Edenius M, Yum J, et al. Halofuginone and other febrifugine derivatives inhibit prolyl-tRNA synthetase. Nat Chem Biol, 2012;8(3):311-7.

\section{Tables}

Table 1 Relationship between glutamyl-prolyl tRNA synthetase (EPRS) mRNA expression and clinicopathological features of patients with hepatocellular carcinoma (HCC) 


\begin{tabular}{|c|c|c|c|c|}
\hline Clinicopathological features & Total number & $\begin{array}{l}\text { Low EPRS mRNA } \\
\text { expression }=274\end{array}$ & $\begin{array}{l}\text { High EPRS mRNA } \\
\text { expression }=96\end{array}$ & $P$ value \\
\hline \multicolumn{5}{|l|}{ Age } \\
\hline$<60$ & 169 & 118 & 51 & 0.0964 \\
\hline$\geq 60$ & 201 & 156 & 45 & \\
\hline \multicolumn{5}{|l|}{ Gender } \\
\hline Male & 249 & 187 & 62 & 0.5288 \\
\hline Female & 121 & 87 & 34 & \\
\hline \multicolumn{5}{|l|}{ TNM stage } \\
\hline$|-| \mid$ & 256 & 206 & 50 & 0.0001 \\
\hline III-IV & 90 & 53 & 37 & \\
\hline \multicolumn{5}{|l|}{ Histopathologic grade } \\
\hline$|-| \mid$ & 232 & 188 & 44 & 0.0002 \\
\hline III-IV & 133 & 83 & 50 & \\
\hline \multicolumn{5}{|l|}{ Tumor size } \\
\hline T1-2 & 276 & 217 & 59 & 0.0010 \\
\hline T3-4 & 94 & 57 & 37 & \\
\hline \multicolumn{5}{|l|}{ Family history } \\
\hline No & 207 & 150 & 57 & 0.5945 \\
\hline Yes & 113 & 85 & 27 & \\
\hline \multicolumn{5}{|l|}{ Vascular invasion } \\
\hline None & 206 & 166 & 40 & 0.0123 \\
\hline Microinvasion & 108 & 73 & 35 & \\
\hline
\end{tabular}

Figures 


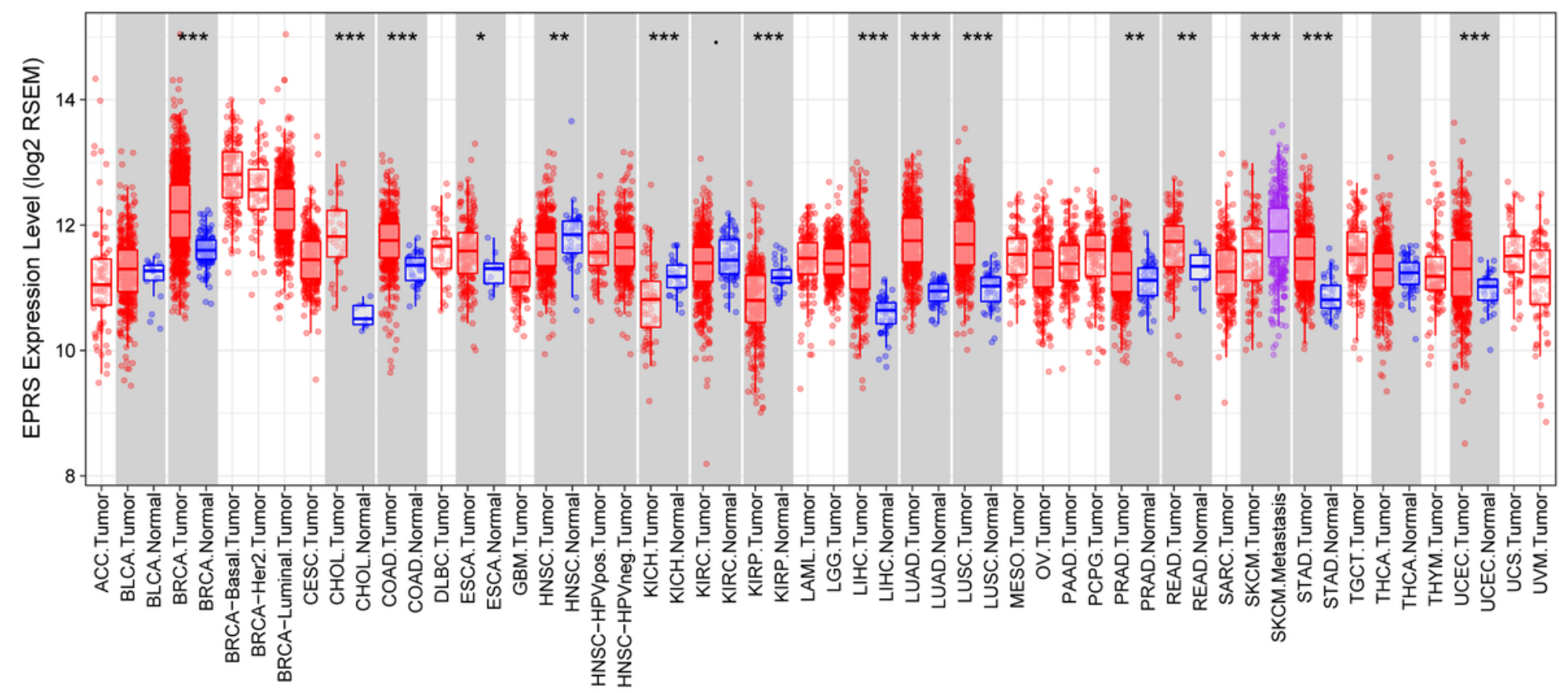

Figure 1

Glutamyl-prolyl tRNA synthetase (EPRS) mRNA expression levels in tumor and adjacent normal tissues based on The Cancer Genome Atlas. Red represents tumor tissues, and blue represents adjacent normal tissues. $\mathrm{P}<0.05$ was considered significant $(* \mathrm{P}<0.05$; $*$ means $\mathrm{P}<0.005$; $* * *$ means $\mathrm{P}<0.0005)$.

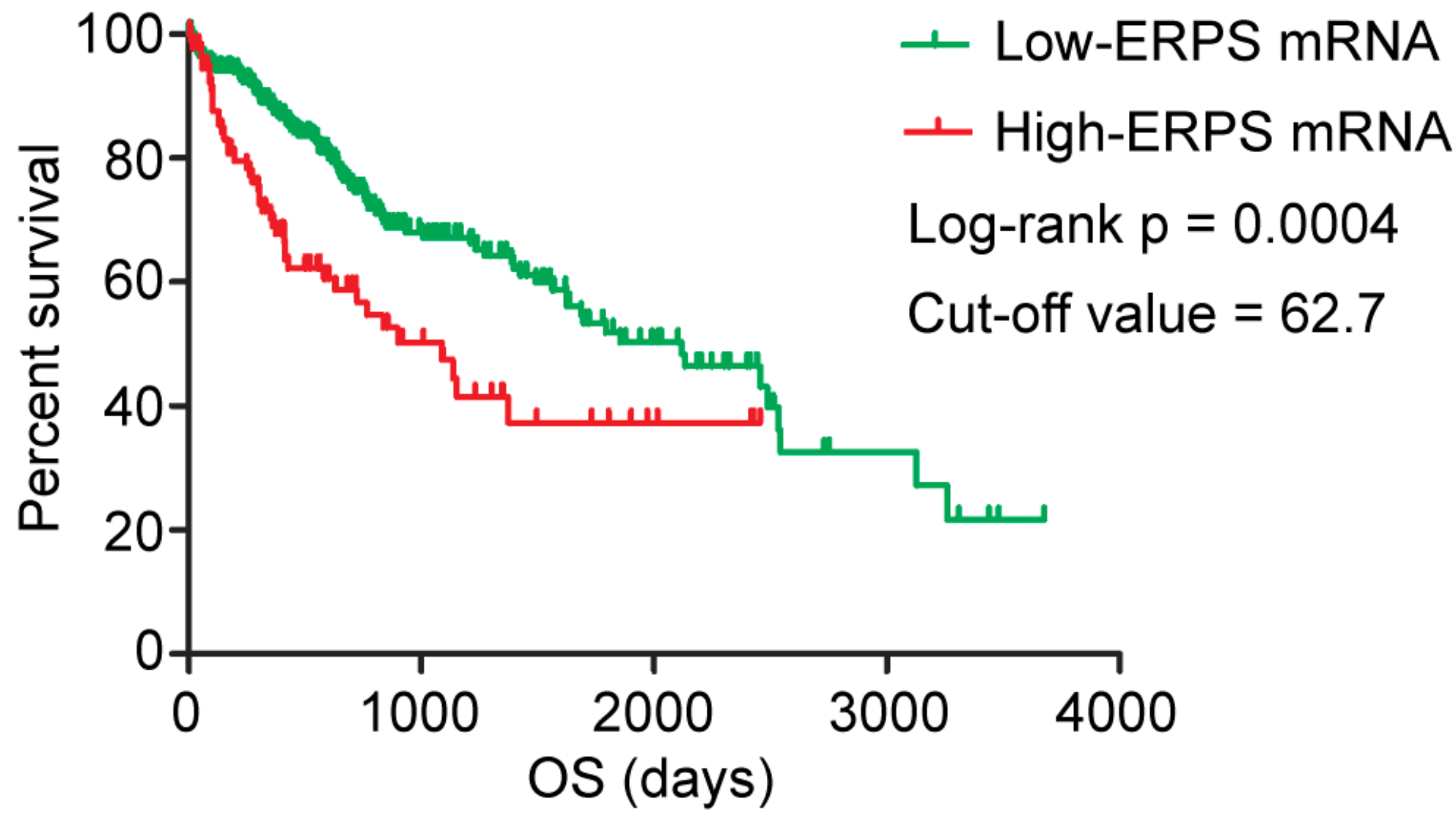

Figure 2 


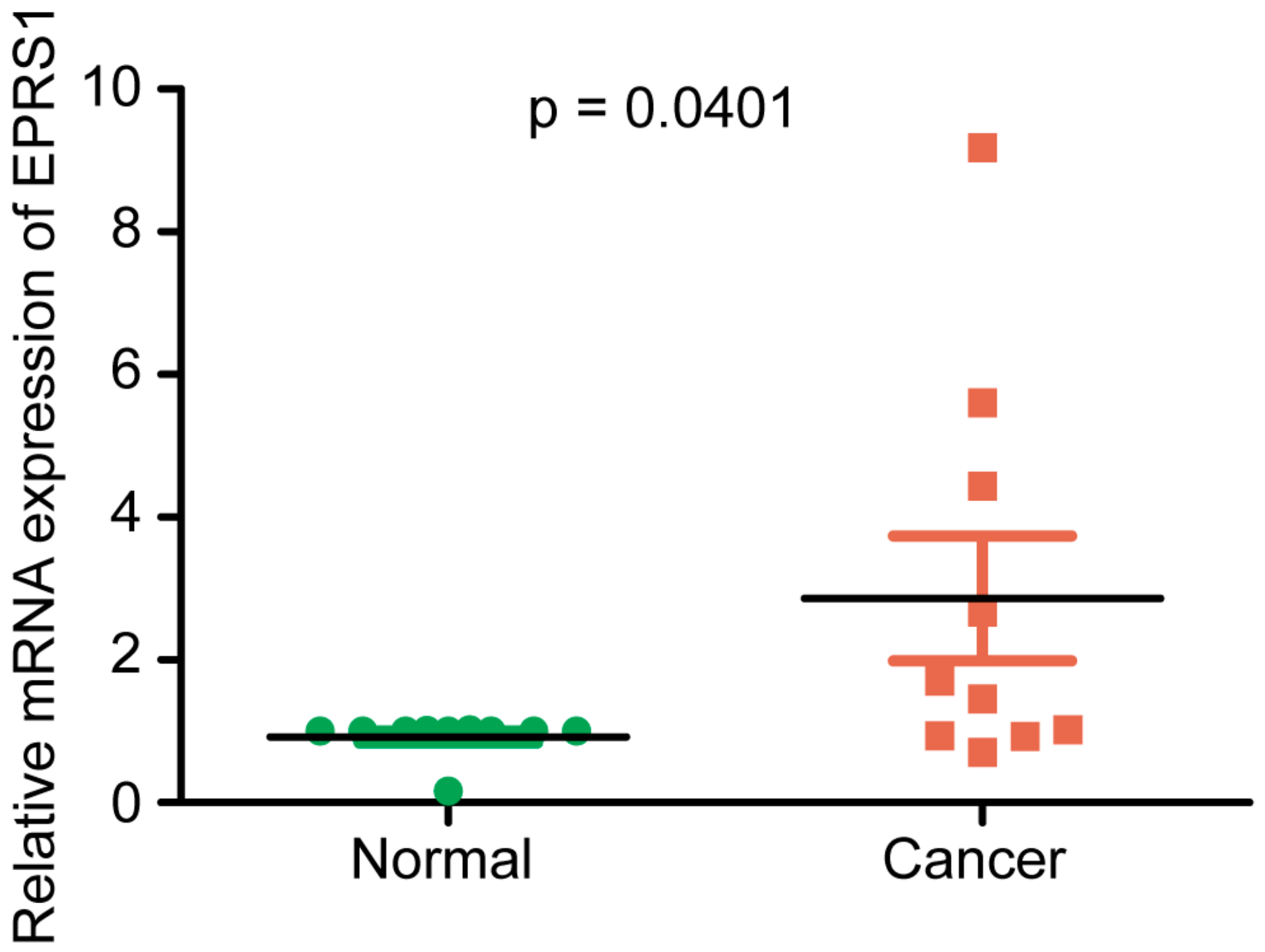

Figure 3

Glutamyl-prolyl tRNA synthetase (EPRS) mRNA is upregulated in hepatocellular carcinoma (HCC) tumor tissues (cancer) compared with adjacent non-tumor tissues (normal). The red color represents the HCC tumor tissue group, and the blue color indicates the adjacent non-tumor tissue group.

\section{Supplementary Files}

This is a list of supplementary files associated with this preprint. Click to download.

- EPRSmRNAexpressiondataandClinicallnformation.xIsx

- EPRSmRNAexpressiondataandSurvivalfollowupdata.xlsx 